\title{
Editorial - Biotechnology special issue
}

\section{Gupta V}

Molecular Glyco-biotechnology Group, Discipline of Biochemistry, National University of Ireland Galway, Ireland Email: vijaifzd@gmail.com; vijai.gupta@nuigalway.ie

Gupta V 2017 Editorial - Biotechnology special issue. Mycosphere 8(3) 360, Doi $10.5943 /$ mycosphere/8/3/1

Fungal systems play an important role in biotechnological applications. Fungal interactions in the environmental are also the main key for producing valuable biomolecules. Their metabolic diversity makes them important bio-resources for a variety of economically valuable industrial and agricultural applications. Knowledge of the biology and physiology of the different taxonomic groups of the fungi from various bio-ecosystems and their associations with the host is the way to understand the successful bioprocess for innovative technological developments.

This special volume in Mycosphere contains eight contribution from experienced researchers in the area of fungal biotechnology, ranging from pathogenicity, VAM symbiosis, to genetic and protein expressions, biological control and industrial applications. 